\title{
ASYMPTOTIC EXPANSIONS OF THE DISTRIBUTIONS OF THE POLYSERIAL CORRELATION COEFFICIENTS
}

\author{
Haruhiko Ogasawara*
}

\begin{abstract}
Asymptotic expansions of the distributions of the sample polyserial correlation coefficients and associated parameter estimators are derived up to order $O(1 / N)$ when the estimators are obtained by full maximum likelihood. The asymptotic results are given under the assumption of multivariate normality for several observed continuous variables and a single unobserved variable underlining the corresponding ordered categorical variable. Asymptotic expansions of the distributions of the pivotal statistics studentized by using the estimate of the information matrix are obtained up to the order next beyond the conventional normal approximation. Numerical examples with simulations are shown in order to illustrate the accuracy of the asymptotic results in finite samples.
\end{abstract}

\section{Introduction}

The polyserial correlation coefficient is the product-moment correlation between two normally distributed variables, where one of the variables is not directly observed, but only the ordered categories, representing consecutive intervals of the unobserved variable, are observed. When the number of the categories is two, the correlation is particularly called the biserial correlation. The biserial and polyserial correlations were introduced by Pearson (1909, 1913), respectively. Soper (1913) obtained the asymptotic standard error (ASE) of Pearson's (1909) estimator of the biserial correlation based on moments. Tate (1955a, b) discussed and gave the maximum likelihood (ML) estimator of the biserial correlation. When there are more than one observed continuous variable, the ML estimators and their ASEs of the (multivariate) biserial correlation coefficients were obtained by Hannan and Tate (1965) (see also Prince \& Tate, 1966). Cox (1974) derived the ASE of the estimator of the polyserial correlation coefficient when there is only one observed continuous variable, using the information matrix.

The point polyserial (biserial) correlation coefficient is the correlation between the observed continuous variable and the categorical variable, where the arbitrary values corresponding to the ordered categories are used for computing the product-moment correlation. Olsson, Drasgow and Dorans (1982) obtained the general relationships between the point and polyserial correlation coefficients. Olsson et al. (1982) also proposed the 2-step limited information estimation of the polyserial correlation, where the threshold parameters, corresponding to the values of the limits of the underlying continuous variable, are estimated by the marginal proportions of the categories.

Key Words and Phrases: polyserial correlation coefficients, Edgeworth expansion, Cornish-Fisher expansion, multivariate normality, thresholds.

* Department of Information and Management Science, Otaru University of Commerce, 3-5-21, Midori, Otaru 047-8501, Japan. E-mail: hogasa@res.otaru-uc.ac.jp 
Lee and Poon (1986) derived the full ML estimators of the polyserial correlation coefficients by using reparameterization when there are more than one observed continuous variable. They derived the ASE estimates based on the observed information matrix, i.e., the negative second derivatives of the log likelihood, which are obtained as byproducts from the Newton-Raphson method for estimating the polyserial correlations. When there are more than one ordered categorical variable, the problem of estimating the correlations between the underlying unobserved continuous variables (the polychoric correlations) occurs. Poon and Lee (1987) dealt with the problem and derived the ML estimators of the polyserial and polychoric correlations. Lee and Poon (1986) also used the part (partition, Poon \& Lee, 1987) ML estimators, where only a subset of the observed variables is used for estimating the parameters.

After these developments, the problem of estimating the parameters in structural models behind the polyserial correlations/covariances (Muthén, 1984; Jöreskog, 1990; Lee, Poon \& Bentler, 1992, 1994, 1995; Muthén \& Satorra, 1995) and the polychoric correlations (Lee, Poon \& Bentler, 1989, 1990a, b; Jöreskog, 1994; Bollen \& MaydeuOlivares, 2007) emerged. In the former set of references, the polychoric correlations are also dealt with as well as the polyserial correlations/covariances. It should be noted that for estimating the structural parameters, mostly the 2-step limited information estimation method is employed, where in the first step, the polyserial and polychoric correlations/covariances are estimated, followed by estimation of the structural parameters typically using the generalized least squares given the estimators of the polyserial and polychoric correlations/covariances. That is, the sampling behavior of the estimators of the structural parameters depends directly on that of the estimators of the polyserial and polychoric correlations/covariances.

The purpose of this study is to obtain the asymptotic expansions of the distributions of the sample polyserial correlation coefficients when there are more than one observed continuous variable and a single ordered categorical variable. The estimators are assumed to be given by full ML involving the reparameterization proposed by Lee and Poon (1986). The asymptotic expansions of the distributions of the estimators studentized by using the sample information matrix are also derived with numerical examples including simulations. The problem of the asymptotic expansions for the sample polychoric correlations is not addressed since the corresponding results have been partially reported by Ogasawara (2010a) in the case of the tetrachoric correlation, i.e., the polychoric correlation when the numbers of the categories of associated categorical variables are limited to 2 .

\section{Formulation of the polyserial correlations}

In this section, the parameterization for the polyserial correlations based on Lee and Poon (1986) is given. Let $\mathbf{X}=\left(X_{1}, \ldots, X_{r}\right)^{\prime}$ be a $r \times 1$ vector representing the observed continuous variables and $Y$ be the unobserved scalar variable underlying the single categorical variable $Z$ that takes values $1,2, \ldots, K$. Suppose that the vector variable $\left(\mathbf{X}^{\prime}, Y\right)^{\prime}$ is multivariate normally distributed as 


$$
\left(\mathbf{X}^{\prime}, Y\right)^{\prime} \sim N\left(\left(\boldsymbol{\mu}^{\prime}, 0\right)^{\prime},\left(\begin{array}{cc}
\boldsymbol{\Sigma} & \boldsymbol{\sigma} \\
\boldsymbol{\sigma}^{\prime} & 1
\end{array}\right)\right)
$$

where $\mathrm{E}\left(\mathbf{X}^{\prime}, Y\right)^{\prime}=\left(\boldsymbol{\mu}^{\prime}, 0\right)^{\prime}, \operatorname{cov}\left(\mathbf{X}^{\prime}, Y\right)^{\prime}=\left(\begin{array}{ll}\boldsymbol{\Sigma} & \boldsymbol{\sigma} \\ \boldsymbol{\sigma}^{\prime} & 1\end{array}\right)$, and $\boldsymbol{\sigma}$ is the $r \times 1$ vector of the population polyserial covariances. The zero mean and unit variance of $Y$ are due to the indeterminacy of the location and dispersion of $Y$. The vector of the population polyserial correlations is denoted by $\boldsymbol{\rho}=(\operatorname{Diag} \boldsymbol{\Sigma})^{-1 / 2} \boldsymbol{\sigma}$, where Diag $(\cdot)$ indicates a diagonal matrix with the diagonal elements being those of the square matrix used as an argument.

The relationships between $Y$ and $Z$ are shown as follows. The probability of $Z=k$ is assumed to be expressed by

$$
\operatorname{Pr}(Z=k)=\operatorname{Pr}\left(\tau_{k-1}<Y \leq \tau_{k}\right)=\Phi\left(\tau_{k}\right)-\Phi\left(\tau_{k-1}\right) \quad(k=1, \ldots, K),
$$

where $\Phi(x)=\int_{-\infty}^{x} \varphi\left(x^{*}\right) d x^{*}, \varphi(x)=(1 / \sqrt{2 \pi}) \exp \left(-x^{2} / 2\right), \tau_{k}$ 's are the population thresholds, $\tau_{0}=-\infty$, and $\tau_{K}=+\infty$. We use the notations $\boldsymbol{\mu}, \boldsymbol{\Sigma}, \tau_{k}$ 's as parameters as well as the population values for simplicity of notation as long as confusion does not occur. Then, the likelihood of the parameters, given $N$ independent observations of $\mathbf{X}$ and $Z$, is

$$
L^{*}=\prod_{i=1}^{N} \exp \left(l_{i}^{*}\right)=\prod_{i=1}^{N} p\left(\mathbf{x}_{i}, z_{i}\right)=\prod_{i=1}^{N} p\left(\mathbf{x}_{i}\right) \operatorname{Pr}\left(z_{i} \mid \mathbf{x}_{i}\right)
$$

where $p\left(\mathbf{x}_{i}\right)=p\left(\mathbf{X}_{i}=\mathbf{x}_{i}\right)=(2 \pi)^{-r / 2}|\boldsymbol{\Sigma}|^{-1 / 2} \exp \left\{-(1 / 2)\left(\mathbf{x}_{i}-\boldsymbol{\mu}\right)^{\prime} \boldsymbol{\Sigma}^{-1}\left(\mathbf{x}_{i}-\boldsymbol{\mu}\right)\right\}$,

$$
\begin{aligned}
& \operatorname{Pr}\left(z_{i} \mid \mathbf{x}_{i}\right)=\operatorname{Pr}\left(Z_{i}=z_{i} \mid \mathbf{X}_{i}=\mathbf{x}_{i}\right)=\operatorname{Pr}\left(\tau_{z_{i}-1}<Y_{i} \leq \tau_{z_{i}} \mid \mathbf{x}_{i}\right)=\Phi_{z_{i}}-\Phi_{z_{i}-1} \equiv \pi_{z_{i}}, \\
& \Phi_{z_{i}}=\Phi\left\{\left(\tau_{z_{i}}-\boldsymbol{\sigma}^{\prime} \boldsymbol{\Sigma}^{-1}\left(\mathbf{x}_{i}-\boldsymbol{\mu}\right)\right) / R\right\}=\Phi\left(\beta_{z_{i}}+\boldsymbol{\xi}^{\prime} \mathbf{x}_{i}\right), \quad R=\left(1-\boldsymbol{\sigma}^{\prime} \boldsymbol{\Sigma}^{-1} \boldsymbol{\sigma}\right)^{1 / 2}, \\
& \beta_{k}=\left(\tau_{k}+\boldsymbol{\sigma}^{\prime} \boldsymbol{\Sigma}^{-1} \boldsymbol{\mu}\right) / R \quad(k=1, \ldots, K-1), \quad \beta_{0}=-\infty, \quad \beta_{K}=+\infty,
\end{aligned}
$$

and $\boldsymbol{\xi}=-\boldsymbol{\Sigma}^{-1} \boldsymbol{\sigma} / R$. The parameters $\beta_{k}$ 's and $\boldsymbol{\xi}$ are the transformed ones. The inverse transformation is expressed by

$$
\tau_{k}=\left(\beta_{k}+\boldsymbol{\xi}^{\prime} \boldsymbol{\mu}\right)\left(1+\boldsymbol{\xi}^{\prime} \boldsymbol{\Sigma} \boldsymbol{\xi}\right)^{-1 / 2} \quad(k=1, \ldots, K-1) \text { and } \boldsymbol{\sigma}=-\boldsymbol{\Sigma} \boldsymbol{\xi}\left(1+\boldsymbol{\xi}^{\prime} \boldsymbol{\Sigma} \boldsymbol{\xi}\right)^{-1 / 2},
$$

where $\left(1+\boldsymbol{\xi}^{\prime} \boldsymbol{\Sigma} \boldsymbol{\xi}\right)^{-1 / 2}=R$.

Using the transformed parameters, the log likelihood becomes

$$
l^{*}=\ln L^{*}=l_{x}^{*}+l_{z}^{*},
$$

where $l_{x}^{*}=-\frac{N}{2} \ln |\boldsymbol{\Sigma}|-\frac{1}{2} \sum_{i=1}^{N}\left(\mathbf{x}_{i}-\boldsymbol{\mu}\right)^{\prime} \boldsymbol{\Sigma}^{-1}\left(\mathbf{x}_{i}-\boldsymbol{\mu}\right)-\frac{N r}{2} \ln (2 \pi)$ and $l_{z}^{*}=\sum_{i=1}^{N} \ln \pi_{z_{i}}$ (for $\pi_{z_{i}}$ see $\left.(2.4)\right)$. Let $\boldsymbol{\theta}=\left(\boldsymbol{\theta}_{x}^{\prime}, \boldsymbol{\theta}_{z}^{\prime}\right)^{\prime}$, where $\boldsymbol{\theta}_{x}=\left(\boldsymbol{\mu}^{\prime}, \mathrm{v}(\boldsymbol{\Sigma})^{\prime}\right)^{\prime}, \boldsymbol{\theta}_{z}=\left(\boldsymbol{\beta}^{\prime}, \boldsymbol{\xi}^{\prime}\right)^{\prime}$, $\boldsymbol{\beta}=\left(\beta_{1}, \ldots, \beta_{K-1}\right)^{\prime}$, and $\mathrm{v}(\cdot)$ is a vectorizing operator taking the non-duplicated elements of a symmetric matrix. Then, from (2.6), we see that the two parameter vectors $\boldsymbol{\theta}_{x}$ and $\boldsymbol{\theta}_{z}$ can be independently estimated by ML. 


\section{Asymptotic expansions}

Let $\bar{l}$ be the mean of the $\log$ likelihood, i.e., $\bar{l}=N^{-1} l^{*}$ (see (2.6)). Denote the ML estimator of $\boldsymbol{\theta}_{0}$ by $\hat{\boldsymbol{\theta}}$, where $\boldsymbol{\theta}_{0}$ is the population parameter vector. The distribution of $\hat{\boldsymbol{\theta}}_{x}=\left(\hat{\boldsymbol{\mu}}^{\prime}, \mathrm{v}(\hat{\boldsymbol{\Sigma}})^{\prime}\right)^{\prime}$ is known, but the results for $\hat{\boldsymbol{\theta}}_{x}$ are included in this section since they will be required in order to obtain the asymptotic expansions of the estimators of the original parameters $\tau_{k}$ 's and $\boldsymbol{\sigma}$ (or $\boldsymbol{\rho}$ ) of interest. From the Taylor series expansion of the first derivatives of $\bar{l}$ about $\boldsymbol{\theta}=\boldsymbol{\theta}_{0}$, we obtain

$$
\begin{aligned}
\frac{\partial \bar{l}}{\partial \hat{\boldsymbol{\theta}}}=\mathbf{0}= & \frac{\partial \bar{l}}{\partial \boldsymbol{\theta}_{0}}+\frac{\partial^{2} \bar{l}}{\partial \boldsymbol{\theta}_{0} \partial \boldsymbol{\theta}_{0}^{\prime}}\left(\hat{\boldsymbol{\theta}}-\boldsymbol{\theta}_{0}\right)+\frac{1}{2} \frac{\partial^{3} \bar{l}}{\partial \boldsymbol{\theta}_{0}\left(\partial \boldsymbol{\theta}_{0}^{\prime}\right)^{<2>}}\left(\hat{\boldsymbol{\theta}}-\boldsymbol{\theta}_{0}\right)^{<2>} \\
& +\frac{1}{6} \frac{\partial^{4} \bar{l}}{\partial \boldsymbol{\theta}_{0}\left(\partial \boldsymbol{\theta}_{0}^{\prime}\right)^{<3>}}\left(\hat{\boldsymbol{\theta}}-\boldsymbol{\theta}_{0}\right)^{<3>}+O_{p}\left(N^{-2}\right),
\end{aligned}
$$

where $\mathbf{x}^{<k>}=\mathbf{x} \otimes \cdots \otimes \mathbf{x}(k$ times of $\mathbf{x})$ is the $k$-fold Kronecker product of $\mathbf{x}$; $\partial \bar{l} / \partial \hat{\boldsymbol{\theta}}=\partial \bar{l} /\left.\partial \boldsymbol{\theta}\right|_{\boldsymbol{\theta}=\hat{\boldsymbol{\theta}}} ; \partial \bar{l} / \partial \boldsymbol{\theta}_{0}=\partial \bar{l} /\left.\partial \boldsymbol{\theta}\right|_{\boldsymbol{\theta}=\boldsymbol{\theta}_{0}}$; and other partial derivatives are defined in similar manners.

General theories for the asymptotic expansions of the distributions of the ML estimators have been developed by using the inverse expansion of $\hat{\boldsymbol{\theta}}$ with respect to the loglikelihood derivatives and associated methods (Bartlett, 1953, Equation (28); Lawley, 1956, Equation (2); Hayakawa, 1977; Akahira \& Takeuchi, 1981, Section 5.2, 1982; Amari, 1985, Section 4.4; McCullagh, 1987, Section 7.3.2; Stafford, 1992; Taniguchi \& Watanabe, 1994; Viraswami \& Reid, 1996, p.268; Taniguchi \& Kakizawa, 2000, Chapter 4; Ogasawara, 2010b). Let $\mathbf{L}=\partial^{2} \bar{l} / \partial \boldsymbol{\theta}_{0} \partial \boldsymbol{\theta}_{0}^{\prime}$ with $\mathrm{E}(\mathbf{L})=\boldsymbol{\Lambda}=-\mathbf{I}$, where $\mathbf{I}$ is the information matrix per observation, and $\mathbf{L}=\boldsymbol{\Lambda}+\mathbf{M}$, where $\mathbf{M}=O_{p}\left(N^{-1 / 2}\right)$. Then, from (3.1), the inverse expansion can be summarized as

$$
\begin{aligned}
& \hat{\boldsymbol{\theta}}-\boldsymbol{\theta}_{0}=\sum_{i=1}^{3} \boldsymbol{\Lambda}^{(i)} \mathbf{l}_{0}^{(i)}+O_{p}\left(N^{-2}\right), \\
& \mathbf{l}_{0}^{(1)} \equiv \frac{\partial \bar{l}}{\partial \boldsymbol{\theta}_{0}}, \mathbf{l}_{0}^{(2)} \equiv\left(\mathrm{v}(\mathbf{M})^{\prime} \otimes \frac{\partial \bar{l}}{\partial \boldsymbol{\theta}_{0}^{\prime}},\left(\frac{\partial \bar{l}}{\partial \boldsymbol{\theta}_{0}^{\prime}}\right)^{<2>}\right)^{\prime}, \\
& \mathbf{l}_{0}^{(3)} \equiv\left[\mathrm{v}(\mathbf{M})^{\prime<2>} \otimes \frac{\partial \bar{l}}{\partial \boldsymbol{\theta}_{0}^{\prime}}, \mathrm{v}(\mathbf{M})^{\prime} \otimes\left(\frac{\partial \bar{l}}{\partial \boldsymbol{\theta}_{0}^{\prime}}\right)^{<2>},\right. \\
&\left.\left\{\frac{\partial^{3} \bar{l}}{\left(\partial \boldsymbol{\theta}_{0}^{\prime}\right)^{<3>}}-\mathrm{E}\left(\frac{\partial^{3} \bar{l}}{\left(\partial \boldsymbol{\theta}_{0}^{\prime}\right)^{<3>}}\right)\right\} \otimes\left(\frac{\partial \bar{l}}{\partial \boldsymbol{\theta}_{0}^{\prime}}\right)^{<2>},\left(\frac{\partial \bar{l}}{\partial \boldsymbol{\theta}_{0}^{\prime}}\right)^{<3>}\right]^{\prime},
\end{aligned}
$$

where $\boldsymbol{\Lambda}^{(i)}=O(1)$ and $\mathbf{l}_{0}^{(i)}=O_{p}\left(N^{-i / 2}\right)(i=1,2,3)$ (for the derivation of (3.2), see Ogasawara, 2012, Section 0). The actual expressions of $\boldsymbol{\Lambda}^{(i)}$ are given in Section A.1 of the appendix.

Let $\theta$ be a generic parameter representing one of the elements of $\boldsymbol{\theta}$ with $\theta_{0}$ and $\hat{\theta}$ similarly defined as before. Let $\lambda^{(i)^{\prime}}$ be the row of $\boldsymbol{\Lambda}^{(i)}(i=1,2,3)$ corresponding to the parameter $\theta$. Then, from (3.2), the first four cumulants of the distribution of $\hat{\theta}$ 
are expressed as follows:

$$
\begin{aligned}
& \mathrm{E}\left(\hat{\theta}-\theta_{0}\right)=N^{-1} \lambda^{(2)^{\prime}} \mathrm{E}\left(N \mathbf{l}_{0}^{(2)}\right)+O\left(N^{-2}\right) \equiv N^{-1} \alpha_{1}+O\left(N^{-2}\right), \\
& \mathrm{E}\left\{(\hat{\theta}-\mathrm{E}(\hat{\theta}))^{2}\right\}=N^{-1} \lambda^{(1)^{\prime}} \mathrm{E}\left(N \mathbf{l}_{0}^{(1)} \mathbf{l}_{0}^{(1)^{\prime}}\right) \lambda^{(1)} \\
& \quad+N^{-2}\left[\lambda^{(2)^{\prime}} \mathrm{E}\left(N^{2} \mathbf{l}_{0}^{(2)} \mathbf{l}_{0}^{(2)^{\prime}}\right) \lambda^{(2)}+2 \lambda^{(1)^{\prime}} \mathrm{E}\left(N^{2} \mathbf{l}_{0}^{(1)} \mathbf{l}_{0}^{(2)^{\prime}}\right) \lambda^{(2)}\right. \\
&\left.\quad+2 \lambda^{(1)^{\prime}} \mathrm{E}\left(N^{2} \mathbf{l}_{0}^{(1)} \mathbf{l}_{0}^{(3)^{\prime}}\right) \lambda^{(3)}-\left\{\lambda^{(2)^{\prime}} \mathrm{E}\left(N \mathbf{l}_{0}^{(2)}\right)\right\}^{2}\right]+O\left(N^{-3}\right) \\
& \equiv N^{-1} \alpha_{2}+N^{-2} \alpha_{\Delta 2}+O\left(N^{-3}\right), \\
& \mathrm{E}\left\{(\hat{\theta}-\mathrm{E}(\hat{\theta}))^{3}\right\} \quad \\
&=N^{-2}\left[\mathrm{E}\left\{N^{2}\left(\lambda^{(1)^{\prime}} \mathbf{l}_{0}^{(1)}\right)^{3}\right\}+3 \mathrm{E}\left\{N^{2}\left(\lambda^{(1)^{\prime}} \mathbf{l}_{0}^{(1)}\right)^{2} \lambda^{(2)^{\prime}} \mathbf{l}_{0}^{(2)}\right\}\right. \\
&\left.\quad-3 \mathrm{E}\left\{N\left(\lambda^{(1)^{\prime}} \mathbf{l}_{0}^{(1)}\right)^{2}\right\} N \mathrm{E}\left(\hat{\theta}-\theta_{0}\right)\right]+O\left(N^{-3}\right) \\
&=N^{-2}\left[\mathrm{E}\left\{N^{2}\left(\lambda^{(1)^{\prime}} \mathbf{l}_{0}^{(1)}\right)^{3}\right\}+3 \mathrm{E}\left\{N^{2}\left(\lambda^{(1)^{\prime}} \mathbf{l}_{0}^{(1)}\right)^{2} \lambda^{(2)^{\prime}} \mathbf{l}_{0}^{(2)}\right\}-3 \alpha_{1} \alpha_{2}\right]+O\left(N^{-3}\right) \\
& \equiv N^{-2} \alpha_{3}+O\left(N^{-3}\right), \\
& \mathrm{E}\left\{(\hat{\theta}-\mathrm{E}(\hat{\theta}))^{4}\right\}-3\left[\mathrm{E}\left\{(\hat{\theta}-\mathrm{E}(\hat{\theta}))^{2}\right\}\right]^{2} \\
&=\mathrm{E}\left\{\left(\hat{\theta}-\theta_{0}\right)^{4}\right\}+N^{-3}\left\{-4 \alpha_{1}\left(\alpha_{3}+3 \alpha_{1} \alpha_{2}\right)+6 \alpha_{1}^{2} \alpha_{2}\right\} \\
& \quad-3 N^{-2} \alpha_{2}^{2}-N^{-3} 6 \alpha_{2} \alpha_{\Delta 2}+O\left(N^{-4}\right) \\
&=\mathrm{E}\left\{\left(\hat{\theta}-\theta_{0}\right)^{4}\right\}-3 N^{-2} \alpha_{2}^{2}-N^{-3}\left(4 \alpha_{1} \alpha_{3}+6 \alpha_{2} \alpha_{\Delta 2}+6 \alpha_{2} \alpha_{1}^{2}\right)+O\left(N^{-4}\right) \\
&= N^{-3}\left[\mathrm{E}\left\{N^{3}\left(\lambda^{(1)^{\prime}} \mathbf{l}_{0}^{(1)}\right)^{4}\right\}+4 \mathrm{E}\left\{N^{3}\left(\lambda^{(1)^{\prime}} \mathbf{l}_{0}^{(1)}\right)^{3} \lambda^{(2)^{\prime}} \mathbf{l}_{0}^{(2)}\right\}\right. \\
&+6 \mathrm{E}\left\{N^{3}\left(\lambda^{(1)^{\prime}} \mathbf{l}_{0}^{(1)}\right)^{2}\left(\lambda^{(2)^{\prime}} \mathbf{l}_{0}^{(2)}\right)^{2}\right\}+4 \mathrm{E}\left\{N^{3}\left(\lambda^{(1)^{\prime}} \mathbf{l}_{0}^{(1)}\right)^{3} \lambda^{(3)^{\prime}} \mathbf{l}_{0}^{(3)}\right\} \\
&\left.\quad-3 N \alpha_{2}^{2}-4 \alpha_{1} \alpha_{3}-6 \alpha_{2} \alpha \Delta 2-6 \alpha_{2} \alpha_{1}^{2}\right]+O\left(N^{-4}\right) \\
& \equiv N^{-3} \alpha_{4}+O\left(N^{-4}\right) .
\end{aligned}
$$

In (3.3), $N^{-1} \alpha_{1}, N^{-1} \alpha_{2}, N^{-2} \alpha_{\Delta 2}, N^{-1 / 2} \alpha_{3} / \alpha_{2}^{3 / 2}$, and $N^{-1} \alpha_{4} / \alpha_{2}^{2}$ are the asymptotic bias, asymptotic variance, added higher-order asymptotic variance, asymptotic skewness, and asymptotic kurtosis, respectively. Using the asymptotic cumulants, from standard statistical theory (e.g., Hall, 1992a), we obtain the following result.

With the assumption of the validity for asymptotic expansion, the cumulative distribution function of $\hat{\theta}$ is expressed by

$$
\begin{aligned}
& \operatorname{Pr}\left(\frac{N^{1 / 2}\left(\hat{\theta}-\theta_{0}\right)}{\alpha_{2}^{1 / 2}} \leq x\right)=\Phi(x)-N^{-1 / 2}\left\{\frac{\alpha_{1}}{\alpha_{2}^{1 / 2}}+\frac{\alpha_{3}}{6 \alpha_{2}^{3 / 2}}\left(x^{2}-1\right)\right\} \varphi(x) \\
& -N^{-1}\left\{\frac{1}{2}\left(\alpha_{\Delta 2}+\alpha_{1}^{2}\right) \frac{x}{\alpha_{2}}+\left(\frac{\alpha_{4}}{24}+\frac{\alpha_{1} \alpha_{3}}{6}\right) \frac{x^{3}-3 x}{\alpha_{2}^{2}}+\frac{\alpha_{3}^{2}\left(x^{5}-10 x^{3}+15 x\right)}{72 \alpha_{2}^{3}}\right\} \varphi(x) \\
& +o\left(N^{-1}\right) .
\end{aligned}
$$

In (3.4), the term $\Phi(x)$ is the conventional normal approximation. The approximations using the terms up to orders $N^{-1 / 2}$ and $N^{-1}$ are the single- and two-term Edgeworth expansions, respectively, where all the terms except $\Phi(x)$ and the residual are counted. In order to obtain the actual results of (3.4), the expectations of the products of the log likelihood derivatives appearing in (3.3) are required, which 
are provided by Ogasawara (2012, Section 1; for the correspondence to $\alpha_{i}$ 's, see the parenthetical notes in the titles of the subsections of the supplement).

Next, the asymptotic expansions of the distributions of the estimators of the original parameters of interest are derived. Let $\boldsymbol{\eta}=\left(\boldsymbol{\tau}^{\prime}, \boldsymbol{\rho}^{\prime}\right)^{\prime}$, where

$$
\begin{aligned}
& \boldsymbol{\tau}=\left(\tau_{1}, \ldots, \tau_{K-1}\right)^{\prime}=\left(\boldsymbol{\beta}+\mathbf{1}_{K-1} \boldsymbol{\xi}^{\prime} \boldsymbol{\mu}\right)\left(1+\boldsymbol{\xi}^{\prime} \boldsymbol{\Sigma} \xi\right)^{-1 / 2}, \\
& \boldsymbol{\rho}=(\operatorname{Diag} \boldsymbol{\Sigma})^{-1 / 2} \boldsymbol{\sigma}=-(\operatorname{Diag} \boldsymbol{\Sigma})^{-1 / 2} \boldsymbol{\Sigma} \xi\left(1+\boldsymbol{\xi}^{\prime} \boldsymbol{\Sigma} \xi\right)^{-1 / 2},
\end{aligned}
$$

and $\mathbf{1}_{K-1}$ is the $(K-1) \times 1$ vector of 1 's. Since (3.5) is a function of $\boldsymbol{\theta}$, say $\boldsymbol{\eta}(\boldsymbol{\theta})$, we define $\hat{\boldsymbol{\eta}}=\boldsymbol{\eta}(\hat{\boldsymbol{\theta}})$ and $\boldsymbol{\eta}_{0}=\boldsymbol{\eta}\left(\boldsymbol{\theta}_{0}\right)$. Then,

$$
\begin{aligned}
\hat{\boldsymbol{\eta}} & =\boldsymbol{\eta}_{0}+\frac{\partial \boldsymbol{\eta}_{0}}{\partial \boldsymbol{\theta}_{0}^{\prime}}\left(\hat{\boldsymbol{\theta}}-\boldsymbol{\theta}_{0}\right)+\frac{1}{2} \frac{\partial^{2} \boldsymbol{\eta}_{0}}{\left(\partial \boldsymbol{\theta}_{0}^{\prime}\right)^{<2>}}\left(\hat{\boldsymbol{\theta}}-\boldsymbol{\theta}_{0}\right)^{<2>}+\frac{1}{6} \frac{\partial^{3} \boldsymbol{\eta}_{0}}{\partial\left(\partial \boldsymbol{\theta}_{0}^{\prime}\right)^{<3>}}\left(\hat{\boldsymbol{\theta}}-\boldsymbol{\theta}_{0}\right)^{<3>}+O_{p}\left(N^{-2}\right) \\
= & \boldsymbol{\eta}_{0}+\left[\frac{\partial \boldsymbol{\eta}_{0}}{\partial \boldsymbol{\theta}_{0}^{\prime}} \boldsymbol{\Lambda}^{(1)} \mathbf{l}_{0}^{(1)}\right]_{O_{p}\left(N^{-1 / 2}\right)}+\left[\frac{\partial \boldsymbol{\eta}_{0}}{\partial \boldsymbol{\theta}_{0}^{\prime}} \boldsymbol{\Lambda}^{(2)} \mathbf{l}_{0}^{(2)}+\frac{1}{2} \frac{\partial^{2} \boldsymbol{\eta}_{0}}{\left(\partial \boldsymbol{\theta}_{0}^{\prime}\right)^{<2>}}\left(\boldsymbol{\Lambda}^{(1)} \mathbf{l}_{0}^{(1)}\right)^{<2>}\right]_{O_{p}\left(N^{-1}\right)} \\
& +\left[\frac{\partial \boldsymbol{\eta}_{0}}{\partial \boldsymbol{\theta}_{0}^{\prime}} \boldsymbol{\Lambda}^{(3)} \mathbf{l}_{0}^{(3)}+\frac{\partial^{2} \boldsymbol{\eta}_{0}}{\left(\partial \boldsymbol{\theta}_{0}^{\prime}\right)^{<2>}}\left\{\left(\boldsymbol{\Lambda}^{(1)} \mathbf{l}_{0}^{(1)}\right) \otimes\left(\boldsymbol{\Lambda}^{(2)} \mathbf{l}_{0}^{(2)}\right)\right\}+\frac{1}{6} \frac{\partial^{3} \boldsymbol{\eta}_{0}}{\left(\partial \boldsymbol{\theta}_{0}^{\prime}\right)^{<3>}}\left(\boldsymbol{\Lambda}^{(1)} \mathbf{l}_{0}^{(1)}\right)^{<3>}\right]_{O_{p}\left(N^{-3 / 2}\right)} \\
& +O_{p}\left(N^{-2}\right),
\end{aligned}
$$

where $[\cdot]_{O_{p}(\cdot)}$ indicates that the sum of the terms in brackets is of order $O_{p}(\cdot)$, and the partial derivatives of $\boldsymbol{\eta}$ with respect to $\boldsymbol{\theta}$ up to the third order are shown in Ogasawara (2012, Section 2). The expressions of (3.6) are summarized as in (3.2):

$$
\hat{\boldsymbol{\eta}}-\boldsymbol{\eta}_{0}=\sum_{i=1}^{3} \mathbf{H}^{(i)} \mathbf{l}_{0}^{(i)}+O_{p}\left(N^{-2}\right),
$$

where $\mathbf{H}^{(i)}=O(1)(i=1,2,3)$,

$$
\begin{aligned}
& \mathbf{H}^{(1)}=\frac{\partial \boldsymbol{\eta}_{0}}{\partial \boldsymbol{\theta}_{0}^{\prime}} \boldsymbol{\Lambda}^{(1)}, \mathbf{H}^{(2)}=\frac{\partial \boldsymbol{\eta}_{0}}{\partial \boldsymbol{\theta}_{0}^{\prime}} \boldsymbol{\Lambda}^{(2)}+\frac{1}{2} \frac{\partial^{2} \boldsymbol{\eta}_{0}}{\left(\partial \boldsymbol{\theta}_{0}^{\prime}\right)^{<2>}} \boldsymbol{\Lambda}^{(1)<2>} \mathbf{I}_{(B, A B)}^{*}, \\
& \mathbf{H}^{(3)}=\frac{\partial \boldsymbol{\eta}_{0}}{\partial \boldsymbol{\theta}_{0}^{\prime}} \boldsymbol{\Lambda}^{(3)}+\frac{\partial^{2} \boldsymbol{\eta}_{0}}{\left(\partial \boldsymbol{\theta}_{0}^{\prime}\right)<2>}\left(\boldsymbol{\Lambda}^{(2)} \otimes \boldsymbol{\Lambda}^{(1)}\right) \mathbf{I}_{(D F, C D E F)}^{*}+\frac{1}{6} \frac{\partial^{3} \boldsymbol{\eta}_{0}}{\left(\partial \boldsymbol{\theta}_{0}^{\prime}\right)^{<3>}} \boldsymbol{\Lambda}^{(1)<3>} \mathbf{I}_{(F, C D E F)}^{*},
\end{aligned}
$$

$\mathbf{I}_{(B, A B)}^{*}$ is the $B \times(A+B)$ matrix consisting of the last $B$ rows of the $(A+B) \times(A+B)$ identity matrix, $\mathbf{I}_{(D F, C D E F)}^{*}$ is the $(D+F) \times(C+D+E+F)$ matrix consisting of the intermediate $D$ rows and the last $F$ rows of the $(C+D+E+F) \times(C+D+E+F)$ identity matrix, and $A, B, \ldots, F$ are the numbers of the elements of the subvectors in $\mathbf{l}_{0}^{(2)}$ and $\mathbf{l}_{0}^{(3)}$ (see (3.2)). Then, from (3.4) and (3.8), we obtain

Theorem 1. The single and two-term Edgeworth expansions of the distributions of $\hat{\boldsymbol{\eta}}$ are obtained by (3.4) and the asymptotic cumulants for $\hat{\boldsymbol{\eta}}$, denoted by $\alpha_{i}^{*}(i=1, \ldots, 4)$ and $\alpha_{\Delta 2}^{*}$, which are given by replacing $\Lambda^{(i)}$ in (3.2) with $\mathbf{H}^{(i)}(i=1,2,3)$ in (3.8) for a generic original parameter $\eta$.

The results in Theorem 1 are useful in order to obtain asymptotic approximations of the distribution of $\hat{\eta}$ as a point estimator of $\eta_{0}$. On the other hand, for testing 
and interval estimation of $\eta_{0}$, the asymptotic expansion of the distribution of the studentized estimator or pivotal statistic shown below is required:

$$
t_{\eta}=N^{1 / 2}\left(\hat{\eta}-\eta_{0}\right) / \hat{\alpha}_{2}^{* 1 / 2},
$$

where $\hat{\alpha}_{2}^{*}$ is the estimator of $\alpha_{2}^{*}=\left\{\mathbf{H}^{(1)} \mathrm{E}\left(\mathbf{l}_{0}^{(1)} \mathbf{l}_{0}^{(1)^{\prime}}\right) \mathbf{H}^{(1)^{\prime}}\right\}_{\eta \eta}=-\left(\mathbf{H}^{(1)} \boldsymbol{\Lambda} \mathbf{H}^{(1)^{\prime}}\right)_{\eta \eta}$ and $(\cdot)_{\eta \eta}$ denotes the diagonal element of the matrix in parentheses corresponding to the parameter $\eta$. The estimator $\hat{\alpha}_{2}^{*}$ can be expanded about $\hat{\alpha}_{2}^{*}=\alpha_{2}^{*}$ as follows:

$$
\hat{\alpha}_{2}^{*}=-\left(\hat{\mathbf{H}}^{(1)} \hat{\mathbf{\Lambda}} \hat{\mathbf{H}}^{(1) \prime}\right)_{\eta \eta}=\alpha_{2}^{*}-\left(\hat{\mathbf{H}}^{(1)} \hat{\mathbf{\Lambda}} \hat{\mathbf{H}}^{(1) \prime}-\mathbf{H}^{(1)} \boldsymbol{\Lambda} \mathbf{H}^{(1)^{\prime}}\right)_{\eta \eta} \equiv \alpha_{2}^{*}+m^{*},
$$

where $m^{*}=O_{p}\left(N^{-1 / 2}\right)$

The statistic $t_{\eta}$ in (3.9) is also expanded about 0 as follows:

$$
t_{\eta}=N^{1 / 2} \alpha_{2}^{*-1 / 2}\left(\boldsymbol{\eta}^{(1)^{\prime}} \mathbf{l}_{0}^{(1)}+\boldsymbol{\eta}^{(2)^{\prime}} \mathbf{l}_{0}^{(2)}\right)-(1 / 2) N^{1 / 2} \alpha_{2}^{*-3 / 2} \boldsymbol{\eta}^{(1)^{\prime}} \mathbf{l}_{0}^{(1)} m^{*}+O_{p}\left(N^{-1}\right),
$$

where $\boldsymbol{\eta}^{(1)^{\prime}}$ and $\boldsymbol{\eta}^{(2)^{\prime}}$ are the rows of $\mathbf{H}^{(1)}$ and $\mathbf{H}^{(2)}$, corresponding to $\eta$, respectively. Then, the first three asymptotic cumulants of $t_{\eta}$, denoted by $\alpha_{i}^{* \prime}(i=1,2,3)$, are obtained from (3.11) as:

$$
\begin{aligned}
\mathrm{E}\left(t_{\eta}\right) & =N^{-1 / 2}\left\{\alpha_{2}^{*-1 / 2} \alpha_{1}^{*}-(1 / 2) \alpha_{2}^{*-3 / 2} \mathrm{E}\left(N \boldsymbol{\eta}^{(1)^{\prime}} \mathbf{l}_{0}^{(1)} m^{*}\right)\right\}+O\left(N^{-3 / 2}\right) \\
& \equiv N^{-1 / 2} \alpha_{1}^{* \prime}+O\left(N^{-3 / 2}\right), \\
\mathrm{E}\left[\left\{t_{\eta}\right.\right. & \left.\left.-\mathrm{E}\left(t_{\eta}\right)\right\}^{2}\right] \equiv 1+O\left(N^{-1}\right) \quad\left(\alpha_{2}^{* \prime}=1\right),
\end{aligned}
$$

and

$$
\begin{aligned}
& \mathrm{E}\left[\left\{t_{\eta}-\mathrm{E}\left(t_{\eta}\right)\right\}^{3}\right]=\mathrm{E}\left(t_{\eta}^{3}\right)-3 \mathrm{E}\left(t_{\eta}\right)+O\left(N^{-3 / 2}\right) \\
& =N^{-1 / 2} \alpha_{2}^{*-3 / 2}\left\{\alpha_{3}^{*}+3 \alpha_{1}^{*}-(9 / 2) \mathrm{E}\left(N \boldsymbol{\eta}^{(1)^{\prime}} \mathbf{l}_{0}^{(1)} m^{*}\right)\right\}-3 N^{-1 / 2} \alpha_{1}^{* \prime}+O\left(N^{-3 / 2}\right) \\
& =N^{-1 / 2}\left\{\alpha_{2}^{*-3 / 2} \alpha_{3}^{*}-3 \alpha_{2}^{*-3 / 2} \mathrm{E}\left(N \boldsymbol{\eta}^{(1)^{\prime}} \mathbf{l}_{0}^{(1)} m^{*}\right)\right\}+O\left(N^{-3 / 2}\right) \\
& \equiv N^{-1 / 2} \alpha_{3}^{* \prime}+O\left(N^{-3 / 2}\right) .
\end{aligned}
$$

The common expectation appearing in $\alpha_{1}^{* \prime}$ and $\alpha_{3}^{* \prime}$ of (3.12a) and (3.12c) is obtained from (3.10) and $\hat{\boldsymbol{\theta}}-\boldsymbol{\theta}_{0}=-\boldsymbol{\Lambda}^{-1} \partial \bar{l} / \partial \boldsymbol{\theta}_{0}+O_{p}\left(N^{-1}\right)$ (see (3.2) and (A1)) as follows:

$$
\begin{aligned}
\mathrm{E}\left\{N\left(\mathbf{l}_{0}^{(1)}\right)_{a} m^{*}\right\} & =-\frac{\partial-\left(\mathbf{H}^{(1)} \boldsymbol{\Lambda} \mathbf{H}^{(1)^{\prime}}\right)_{\eta \eta}}{\partial \boldsymbol{\theta}_{0}^{\prime}} \boldsymbol{\Lambda}^{-1} \mathrm{E}\left\{N \frac{\partial \bar{l}}{\partial \boldsymbol{\theta}_{0}} \frac{\partial \bar{l}}{\partial\left(\boldsymbol{\theta}_{0}\right)_{a}}\right\}+O\left(N^{-1}\right) \\
& =-\frac{\partial\left(\mathbf{H}^{(1)} \boldsymbol{\Lambda} \mathbf{H}^{(1)^{\prime}}\right)_{\eta \eta}}{\partial\left(\boldsymbol{\theta}_{0}\right)_{a}}+O\left(N^{-1}\right) \\
& =\left(-2 \frac{\partial \mathbf{H}^{(1)}}{\partial\left(\boldsymbol{\theta}_{0}\right)_{a}} \boldsymbol{\Lambda} \mathbf{H}^{(1)^{\prime}}-\mathbf{H}^{(1)} \frac{\partial \boldsymbol{\Lambda}}{\partial\left(\boldsymbol{\theta}_{0}\right)_{a}} \mathbf{H}^{(1)^{\prime}}\right)_{\eta \eta}+O\left(N^{-1}\right) \quad(a=1, \ldots, q)
\end{aligned}
$$

where $q$ is the number of parameters; from the Bartlett identity (see (A3))

$$
\frac{\partial \boldsymbol{\Lambda}}{\partial\left(\boldsymbol{\theta}_{0}\right)_{a}}=\mathrm{E}\left(\frac{\partial^{3} \bar{l}}{\partial \boldsymbol{\theta}_{0} \partial \boldsymbol{\theta}_{0}^{\prime} \partial\left(\boldsymbol{\theta}_{0}\right)_{a}}\right)+\mathrm{E}\left(\frac{\partial^{2} l_{i}^{*}}{\partial \boldsymbol{\theta}_{0} \partial \boldsymbol{\theta}_{0}^{\prime}} \frac{\partial l_{i}^{*}}{\partial\left(\boldsymbol{\theta}_{0}\right)_{a}}\right) ;
$$


the expectations on the right-hand side of the last equation are provided by Ogasawara (2012, Section 1);

$$
\frac{\partial \mathbf{H}^{(1)}}{\partial\left(\boldsymbol{\theta}_{0}\right)_{a}}=\frac{\partial^{2} \boldsymbol{\eta}_{0}}{\partial \boldsymbol{\theta}_{0}^{\prime} \partial\left(\boldsymbol{\theta}_{0}\right)_{a}} \boldsymbol{\Lambda}^{(1)}+\frac{\partial \boldsymbol{\eta}_{0}}{\partial \boldsymbol{\theta}_{0}^{\prime}} \frac{\partial \boldsymbol{\Lambda}^{(1)}}{\partial\left(\boldsymbol{\theta}_{0}\right)_{a}},
$$

where $\partial^{2} \boldsymbol{\eta}_{0} / \partial \boldsymbol{\theta}_{0}^{\prime} \partial\left(\boldsymbol{\theta}_{0}\right)_{a}$ is shown in Ogasawara (2012, Section 2); and

$$
\frac{\partial \boldsymbol{\Lambda}^{(1)}}{\partial\left(\boldsymbol{\theta}_{0}\right)_{a}}=-\frac{\partial \boldsymbol{\Lambda}^{-1}}{\partial\left(\boldsymbol{\theta}_{0}\right)_{a}}=\boldsymbol{\Lambda}^{-1} \frac{\partial \boldsymbol{\Lambda}}{\partial\left(\boldsymbol{\theta}_{0}\right)_{a}} \boldsymbol{\Lambda}^{-1} .
$$

Let $\hat{\alpha}_{1}^{* \prime}$ and $\hat{\alpha}_{3}^{* \prime}$ be the sample counterparts of $\alpha_{1}^{* \prime}$ and $\alpha_{3}^{* \prime}$ using $\hat{\boldsymbol{\eta}}$ and $\hat{\boldsymbol{\theta}}$ in place of $\boldsymbol{\eta}_{0}$ and $\boldsymbol{\theta}_{0}$, respectively. Then, from the standard statistical theory (see Hall, 1992a), we obtain

Theorem 2. The confidence interval of $\eta_{0}$ with the asymptotic confidence coefficient $1-\tilde{\alpha}$ (e.g., $\tilde{\alpha}=0.05)$ accurate up to order $O\left(N^{-1 / 2}\right)$ based on the Cornish-Fisher expansion is

$$
\hat{\eta}+\left[ \pm z_{\tilde{\alpha} / 2}-N^{-1 / 2}\left\{\hat{\alpha}_{1}^{* \prime}+\left(\hat{\alpha}_{3}^{* \prime} / 6\right)\left(z_{\tilde{\alpha} / 2}^{2}-1\right)\right\}\right] n^{-1 / 2} \hat{\alpha}_{2}^{* 1 / 2},
$$

where $1-\tilde{\alpha}=\int_{-\infty}^{z_{\tilde{\alpha}}} \varphi\left(z^{*}\right) d z^{*}$.

It is known that the approximate cumulative distribution function for $\hat{\eta}$ obtained by the single-term Edgeworth expansion corresponding to the above expansion can be locally decreasing. Such anomalous phenomena can be avoided by the method involving variable transformation proposed by Hall (1992b), although the asymptotic accuracy by Hall's method is the same as that of (3.17). The confidence interval by Hall is expressed as

$$
\begin{aligned}
& \hat{\eta}-N^{-1} \hat{\alpha}_{2}^{* 1 / 2} \hat{\alpha}_{1}^{* \prime} \\
& +6 \hat{\alpha}_{2}^{* 1 / 2}\left(\hat{\alpha}_{3}^{* \prime}\right)^{-1}\left[\left\{1-(1 / 2) \hat{\alpha}_{3}^{* \prime}\left( \pm N^{-1 / 2} z_{\tilde{\alpha} / 2}-\left(N^{-1} / 6\right) \hat{\alpha}_{3}^{* \prime}\right)\right\}^{1 / 3}-1\right] .
\end{aligned}
$$

So far, the pivotal statistic is obtained by the sample information matrix (see $(3.10))$. As mentioned earlier, Lee and Poon (1986) used $\partial^{2} \bar{l} / \partial \hat{\boldsymbol{\theta}} \partial \hat{\boldsymbol{\theta}}^{\prime}(\equiv \hat{\mathbf{L}})$ in place of $\hat{\boldsymbol{\Lambda}}(=-\hat{\mathbf{I}})$ in order to have the ASE estimates. When $\hat{\mathbf{L}}$ is used, it can be shown similarly as Ogasawara (2010b) that the asymptotic cumulants of the modified studentized estimators up to the third order are the same as $\alpha_{i}^{* \prime}(i=1,2,3)$ obtained earlier (for the derivation, see Section A.2 of the appendix).

\section{Numerical examples}

In this section, numerical examples of the asymptotic expansions for the nonstudentized and studentized estimators up to order $O\left(N^{-1 / 2}\right)$ are shown. Three sets of artificial data (Cases 1, 2, and 3) are used. The population values of the parameters are shown in Table 1 . Case 1 has 2 observed continuous variables $(r=2)$ and 4 categories $(K=4)$ in a single categorical variable, where $\boldsymbol{\mu}=(1,1)^{\prime}, \boldsymbol{\Sigma}=\left(\begin{array}{ll}4 & 0 \\ 0 & 4\end{array}\right)$, and 
Table 1: Simulated and asymptotic cumulants of the non-studentized parameter estimators

\begin{tabular}{|c|c|c|c|c|c|c|c|c|c|c|c|c|}
\hline $\begin{array}{c}\text { Population } \\
\text { parameter } \\
\text { values }\end{array}$ & $\begin{array}{r}\alpha \\
(50) \\
\end{array}$ & $\begin{array}{r}{ }^{1 / 2}(\mathrm{C} \\
{ }^{1 / 2} \mathrm{Sin} \\
(200\end{array}$ & St & A & $(50)$ & $\begin{array}{l}\alpha_{1} \\
\mathrm{Si}\end{array}$ & s) & & & $\begin{array}{r}\alpha_{2}^{3 /} \\
\mathrm{Si} \\
(20 \\
\end{array}$ & & Asy. \\
\hline $\begin{array}{c}\text { Case } 1 \\
\tau_{1}=-1 \\
\tau_{2}=0 \\
\tau_{3}=1 \\
\rho_{1}=.3 \\
\rho_{2}=.3 \\
\end{array}$ & & $\begin{array}{l}1.533 \\
1.255 \\
1.526 \\
.9818 \\
.9817 \\
\end{array}$ & & & $\begin{array}{r}-1.32 \\
.04 \\
1.31 \\
-.11 \\
-.11 \\
\end{array}$ & $\begin{array}{r}-1.14 \\
.15 \\
1.37 \\
-.09 \\
-.03 \\
\end{array}$ & $\begin{array}{r}1 \\
- \\
\end{array}$ & $\begin{array}{r}-1.16 \\
0 \\
1.16 \\
-.03 \\
-.03 \\
\end{array}$ & $\begin{array}{r}-3.48 \\
.10 \\
3.65 \\
-1.79 \\
-1.76 \\
\end{array}$ & $\begin{array}{r}-2.88 \\
-.14 \\
2.71 \\
-1.55 \\
-1.53 \\
\end{array}$ & $\begin{array}{r}-2.82 \\
.19 \\
2.81\end{array}$ & $\begin{array}{r}-1.95 \\
0\end{array}$ \\
\hline $\begin{array}{c}\text { Case } 2 \\
\tau_{1}=-1 \\
\tau_{2}=0 \\
\tau_{3}=1 \\
\rho_{1}=.3\end{array}$ & & $\begin{array}{l}1.528 \\
1.254 \\
1.529 \\
.9771 \\
\end{array}$ & & & $\begin{array}{c}1 . \\
-.\end{array}$ & $\begin{array}{r}-1.11 \\
.19 \\
1.23 \\
-.03 \\
\end{array}$ & 1 & 15 & $\begin{array}{r}-3.48 \\
-.07 \\
3.63 \\
-1.73 \\
\end{array}$ & $\begin{array}{r}-2.81 \\
.02 \\
2.97 \\
-1.87 \\
\end{array}$ & & $\begin{array}{r}-2.32 \\
0 \\
2.32 \\
-.89 \\
\end{array}$ \\
\hline $\begin{array}{c}\text { Case } 3 \\
\tau_{1}=-.7 \\
\tau_{2}=.3 \\
\rho_{1}=.2 \\
\rho_{2}=.6\end{array}$ & $\begin{array}{r}1.416 \\
1.295 \\
1.072 \\
.736 \\
\end{array}$ & $\begin{array}{r}1.377 \\
1.266 \\
1.082 \\
.760 \\
\end{array}$ & & & $\begin{array}{r}.28 \\
-.48 \\
-.47 \\
\end{array}$ & $\begin{array}{r}.41 \\
-.12 \\
-.02 \\
\end{array}$ & $\begin{array}{l}- \\
- \\
-\end{array}$ & & $\begin{array}{r}-2.03 \\
.89 \\
-1.25 \\
-4.24 \\
\end{array}$ & $\begin{array}{r}-1.75 \\
.63 \\
-.87 \\
-3.06 \\
\end{array}$ & - & $\begin{array}{r}-.62 \\
.21 \\
-.36 \\
-1.08 \\
\end{array}$ \\
\hline
\end{tabular}

Note. Sim.: Simulated values, Asy.: Asymptotic values.

the population threshold parameters are symmetrically distributed. Case 2 with $r=$ 1 and $K=4$ represents a subproblem dealing with a subset of variables in Case 1 , where the first or second observed continuous variable is omitted. That is, Case 2 was employed for the part or partition ML estimation in Case 1. Generally, the part or partition estimation is repeated as many as the number of observed continuous variables. However, in Case 1, the 2 observed variables are exchangeable. So, only one set of results is shown. In Case 3 with $r=2$ and $K=3$, the population values of the threshold parameters are asymmetrically distributed, where $\boldsymbol{\mu}=(1,1)^{\prime}, \boldsymbol{\Sigma}=\left(\begin{array}{ll}4 & 0 \\ 0 & 4\end{array}\right)$ with different population polyserial correlations.

In Table 1, the asymptotic cumulants independent of $N$ for the non-studentized estimators are shown, where the expectations (of the products) of the log likelihood derivatives are obtained by the numerical integration using the Gaussian quadrature with 10 points (Stroud \& Secrest, 1966; for actual computation, see Ogasawara, 2012, Section 3). In Cases 1 and 3 with 2 observed continuous variables, $10^{2}$ mesh points for the Gaussian quadrature are used. The computation using more quadrature points was carried out. However, the results were almost the same.

The table also lists the simulated cumulants obtained by the simulations with $10^{5}$ replications each for 3 sample sizes 50, 200 and 800. In a replication, a set of parameter estimates was obtained by ML using the Newton-Raphson iteration. In some replications when $N=50$ and 200, we had difficulties in estimating the parameters or associated values due to zero frequencies in the categories of the discrete variable and the degenerate values of the estimated sample cumulants. These samples were excluded until regular $10^{5}$ sets of estimates were available. The numbers of excluded 
Table 2: Simulated and asymptotic cumulants of the studentized parameter estimators

\begin{tabular}{|c|c|c|c|c|c|c|c|c|c|c|c|c|}
\hline $\begin{array}{c}\text { Population } \\
\text { parameter } \\
\text { values }\end{array}$ & $\begin{array}{r}\left(\alpha_{2}^{* \prime}\right. \\
(50) \\
\end{array}$ & $\begin{array}{r})^{1 / 2} \\
\text { Sim } \\
(200) \\
\end{array}$ & 0 & Asy. & 50) & $\begin{array}{r}\alpha_{1}^{\prime} \\
\mathrm{S} \\
(20 \\
\end{array}$ & s) & $\mathrm{F}$ & 50) & $\begin{array}{r}\alpha_{3}^{* \prime} \\
\mathrm{S} \\
(20 \\
\end{array}$ & $\begin{array}{l}\text { less) } \\
\text { J) } \\
00)\end{array}$ & Asy. \\
\hline $\begin{array}{c}\text { Case } 1 \\
\tau_{1}=-1 \\
\tau_{2}=0 \\
\tau_{3}=1 \\
\rho_{1}=.3 \\
\rho_{2}=.3\end{array}$ & $\begin{array}{l}.988 \\
.998\end{array}$ & $\begin{array}{r}1.001 \\
1.001 \\
.996 \\
1.024 \\
1.024\end{array}$ & & 1 & $\begin{array}{r}-.20 \\
.03 \\
.19 \\
.53 \\
.53\end{array}$ & $\begin{array}{r}-.16 \\
.12\end{array}$ & $\begin{array}{r}-.16 \\
.05\end{array}$ & .26 & $\begin{array}{r}1.14 \\
.08 \\
-1.01 \\
2.65 \\
2.45\end{array}$ & $\begin{array}{r}.82 \\
-.14 \\
-.97 \\
2.37 \\
2.32 \\
\end{array}$ & & $\begin{array}{r}1.10 \\
0 \\
1.10\end{array}$ \\
\hline $\begin{array}{c}\text { Case } 2 \\
\tau_{1}=-1 \\
\tau_{2}=0 \\
\tau_{3}=1 \\
\rho_{1}=.3\end{array}$ & $\begin{array}{r}.988 \\
.999 \\
.988 \\
1.088 \\
\end{array}$ & $\begin{array}{r}.997 \\
.998 \\
.997 \\
1.018 \\
\end{array}$ & 1.005 & & $\begin{array}{r}-.14 \\
.03 \\
.16 \\
.61 \\
\end{array}$ & $\begin{array}{r}-.14 \\
.15 \\
.22 \\
.58 \\
\end{array}$ & $\begin{array}{r}-.14 \\
.16 \\
.31 \\
.49 \\
\end{array}$ & $\begin{array}{r}-.22 \\
0 \\
.22 \\
.44 \\
\end{array}$ & & $\begin{array}{r}.0 \\
-.7 \\
1.9 \\
\end{array}$ & & $\begin{array}{r}.92 \\
0 \\
-.92 \\
1.97 \\
\end{array}$ \\
\hline $\begin{array}{c}\text { Case } \\
\tau_{1}= \\
\tau_{2}= \\
\rho_{1}= \\
\rho_{2}=\end{array}$ & $\begin{array}{l}.995 \\
.001 \\
.057 \\
.986\end{array}$ & $\begin{array}{r}.998 \\
.994 \\
1.023 \\
1.032\end{array}$ & 1.009 & 1 & $\begin{array}{r}-.28 \\
.06 \\
-.06 \\
.50\end{array}$ & $\begin{array}{r}.17 \\
.30 \\
1.25\end{array}$ & $\begin{array}{r}-.20 \\
.15 \\
.41 \\
1.18\end{array}$ & $\begin{array}{r}.11 \\
.36 \\
1.08\end{array}$ & $\begin{array}{r}.69 \\
-.13 \\
1.26 \\
1.88\end{array}$ & $\begin{array}{r}-.29 \\
1.77 \\
5.03\end{array}$ & 4.33 & $\begin{array}{r}1.05 \\
-.42 \\
2.00 \\
5.52\end{array}$ \\
\hline
\end{tabular}

Note. Sim.: Simulated values; Asy.: Asymptotic values.

samples when $N=50$ (200) are 719 (1) for Case 1 and 26 (0) for Case 2, and 5049 (15) for Case 3. When $N=800$, no samples were deleted.

From the regular $10^{5}$ estimates for each parameter, the simulated cumulants were calculated from $k$-statistics (unbiased estimators of the population cumulants; see e.g., Stuart \& Ort, 1994, Section 12.10) multiplied by $N$ raised to appropriate powers so as to be comparable to the corresponding asymptotic values independent of $N$. In Table 1, the simulated values are, as a whole, reasonably similar to the corresponding asymptotic values. We can see that the simulated and asymptotic biases for the polyserial correlations are relatively small while the biases for the thresholds are positive (negative) for the positive (negative) population values. We also find that the results of Case 2 are similar to those of Case 1.

Table 2 shows the similar results for the studentized estimators. The absolute biases of the threshold estimators relative to the corresponding asymptotic standard errors have been reduced from the results in Table 1 while those for the polyserial correlations have somewhat increased. Further, the simulated and asymptotic skewnesses of the polyserial correlations are positive and relatively large. Again, the results of Case 2 are similar to those of Case 1 in Table 2.

In Table 3 , the $10^{5} \times$ root mean square errors of the asymptotic cumulative distribution functions for the studentized estimators obtained by the single-term Edgeworth expansion (E1) and Hall's (1992b) method involving variable transformation are shown (for the distribution function by Hall, 1992b, see Ogasawara, 2008). The true distribution functions were obtained by the simulation of $10^{5}$ replications as in Tables 1 and 2. An error is defined as the asymptotic value minus the corresponding simulated value. The errors were evaluated at the 40 equally spaced points from -3.8 
Table $3: 10^{5} \times$ root mean square errors of the asymptotic distribution functions of the studentized estimators

\begin{tabular}{crrrrrrrrrr}
\hline \multicolumn{1}{c}{$(N)$} & \multicolumn{3}{c}{$(50)$} & \multicolumn{4}{c}{$(200)$} & & \multicolumn{2}{c}{$(800)$} \\
Case & & $\mathrm{N}^{*}$ & $\mathrm{E} 1$ & Hall & $\mathrm{N}^{*}$ & $\mathrm{E} 1$ & Hall & $\mathrm{N}^{*}$ & $\mathrm{E} 1$ & Hall \\
\hline 1 & $\tau_{1}$ & 1016 & 950 & 959 & 135 & 576 & 576 & 166 & 101 & 102 \\
& $\tau_{2}$ & 253 & 253 & 253 & 186 & 186 & 186 & 51 & 51 & 51 \\
& $\tau_{3}$ & 976 & 948 & 957 & 225 & 372 & 374 & 211 & 85 & 86 \\
& $\rho_{1}$ & 1298 & 742 & 720 & 594 & 250 & 245 & 357 & 150 & 151 \\
& $\rho_{2}$ & 1279 & 691 & 671 & 663 & 306 & 307 & 280 & 69 & 68 \\
\hline \multirow{2}{2}{2} & $\tau_{1}$ & 1268 & 1425 & 1431 & 359 & 738 & 738 & 168 & 165 & 166 \\
& $\tau_{2}$ & 564 & 564 & 564 & 206 & 206 & 206 & 111 & 111 & 111 \\
& $\tau_{3}$ & 1276 & 1402 & 1408 & 338 & 698 & 699 & 245 & 96 & 97 \\
& $\rho_{1}$ & 1439 & 797 & 773 & 656 & 264 & 260 & 274 & 70 & 69 \\
\hline 3 & $\tau_{1}$ & 1079 & 580 & 580 & 239 & 243 & 243 & 176 & 73 & 74 \\
& $\tau_{2}$ & 278 & 322 & 320 & 293 & 116 & 116 & 119 & 75 & 76 \\
& $\rho_{1}$ & 677 & 1060 & 1049 & 396 & 241 & 239 & 231 & 108 & 109 \\
& $\rho_{2}$ & 1186 & 1283 & 1412 & 1365 & 453 & 454 & 642 & 195 & 196 \\
\hline
\end{tabular}

Note. $N^{*}$ : Normal approximation; E1: Single-term Edgeworth expansion; Hall: Hall's method involving variable transformation.

to 4.0. The errors were squared and averaged over the 40 points, followed by taking its square root. In Table 3 , the results of the conventional normal approximation $\left(\mathrm{N}^{*}\right)$ are also shown for comparison. The equal values for the central threshold $\tau_{2}$ in Cases 1 and 2 over $\mathrm{N}^{*}, \mathrm{E} 1$ and Hall are due to $\alpha_{1}^{* \prime}=\alpha_{3}^{* \prime}=0$. The table shows that when $N=50$ and 200, the results by E1 and Hall are poor depending on Cases 1, 2 and 3 . However, when $N=800$, the errors by E1 and Hall, which are similar, are smaller than those by the normal approximation $\left(\mathrm{N}^{*}\right)$.

Table 4 illustrates the results of the replications of interval estimation by $\mathrm{N}^{*}$ and Hall for selected parameters using the same data set as used earlier when $N=800$. The table shows the proportions of the population parameters lower than the endpoints of the confidence intervals, which were obtained from $10^{5}$ simulated one-tailed confidence intervals. As is expected from Table 3, the results of $N=50$ and 200 are poor and are not shown in the table. From Table 4 we find that Hall gives proportions more similar to the nominal values than $\mathrm{N}^{*}$ especially for the serial correlations.

\section{Some remarks}

In previous sections, the asymptotic results depend on the multivariate normality of $\left(\mathbf{X}^{\prime}, Y\right)^{\prime}$. When the normality assumption is violated, different model formulations are required (see e.g., Kraemer, 1981) although investigation of robustness issues under nonnormality is also important. When the distribution of $\mathbf{X}$ is known and when the distribution of unobservable $Y$ can be seen as normal, conditional on $\mathbf{X}$, similar results can be obtained by replacing $p\left(\mathbf{x}_{i}\right)$ based on the normality in (2.4) by the corresponding non-normal expression. When $\mathbf{x}_{i}$ 's are seen as fixed values, a simpler model without $\boldsymbol{\theta}_{x}$ can be used as long as the conditional distributions of $Y$ given $\mathbf{x}_{i}$ 's 
Table 4: Simulated proportions lower than the endpoints of the confidence intervals

\begin{tabular}{rrrrrrrrr}
\hline \multicolumn{6}{c}{$N=800$} & \multicolumn{6}{c}{ Nominal values } \\
& & .0050 & .0250 & .1000 & .5000 & 9000 & .9750 & .9950 \\
\hline Case 1 & $\tau_{1} \mathrm{~N}^{*}$ & .0053 & .0256 & .0992 & .4958 & .8999 & .9752 & .9952 \\
& Hall & .0049 & .0250 & .1001 & .5026 & .9009 & .9745 & .9947 \\
& $\tau_{2} \mathrm{~N}^{*}$ & .0051 & .0258 & .1008 & .5003 & .9005 & .9751 & .9951 \\
& Hall & .0051 & .0258 & .1007 & .5003 & .9006 & .9751 & .9951 \\
& $\rho_{1} \mathrm{~N}^{*}$ & .0067 & .0290 & .1071 & .5061 & .9044 & .9775 & .9960 \\
& Hall & .0045 & .0248 & .1016 & .5050 & .9004 & .9748 & .9949 \\
\hline Case 2 & $\tau_{1} \mathrm{~N}^{*}$ & .0053 & .0257 & .0983 & .4977 & .9010 & .9758 & .9957 \\
& Hall & .0049 & .0256 & .0991 & .5043 & .9019 & .9747 & .9952 \\
& $\tau_{2} \mathrm{~N}^{*}$ & .0050 & .0248 & .1004 & .5032 & .9003 & .9760 & .9952 \\
& Hall & .0050 & .0248 & .1003 & .5032 & .9004 & .9760 & .9951 \\
& $\rho_{1} \mathrm{~N}^{*}$ & .0067 & .0282 & .1050 & .5009 & .9039 & .9768 & .9957 \\
& Hall & .0049 & .0245 & .0999 & .4996 & .9003 & .9748 & .9951 \\
\hline Case 3 3 & $\tau_{1} \mathrm{~N}^{*}$ & .0049 & .0249 & .0996 & .4956 & .8991 & .9750 & .9954 \\
& Hall & .0047 & .0244 & .1004 & .5014 & .8999 & .9743 & .9950 \\
& $\tau_{2} \mathrm{~N}^{*}$ & .0050 & .0244 & .1004 & .5019 & .9019 & .9758 & .9951 \\
& Hall & .0052 & .0246 & .1000 & .4991 & .9015 & .9759 & .9952 \\
& $\rho_{1} \mathrm{~N}^{*}$ & .0065 & .0274 & .1050 & .5029 & .9019 & .9768 & .9956 \\
& Hall & .0046 & .0235 & .1003 & .5025 & .8992 & .9751 & .9951 \\
& $\rho_{2} \mathrm{~N}^{*}$ & .0085 & .0327 & .1111 & .5077 & .9085 & .9804 & .9970 \\
& Hall & .0041 & .0238 & .1001 & .5053 & .8991 & .9743 & .9949 \\
\hline
\end{tabular}

Note. $\mathrm{N}^{*}$ : Normal approximation; Hall: Hall's method involving variable transformation.

are normal.

The results of Case 2 in the numerical examples are encouraging in that the asymptotic cumulants up to the third order are almost the same as those of Case 1 in practical sense. An advantage of the part/partition ML method may be the use of the unidimensional Gaussian quadrature, which gives results more stable than those of the multidimensional cases. Another possible advantage for the part/partition ML method might be found in the weaker assumption of normality for subsets of original variables.

Throughout this paper, the validity of the asymptotic expansion was assumed. This is because the situation for the polyserial correlations is different from the standard one with all observable variables being continuous (see e.g., Bhattacharya \& Gosh, 1978). However, an anonymous reviewer gave a conjecture on the validity of the asymptotic expansion in this paper with an associated reference (Jensen, 1989). The author is indebted to the reviewer for the promising result.

\section{Acknowledgements}

This work was partially supported by a Grant-in-Aid for Scientific Research from the Japanese Ministry of Education, Culture, Sports, Science and Technology No.20500341. 


\section{Appendix}

A.1 Expressions of $\boldsymbol{\Lambda}^{(i)}(i=1,2,3)$ in (3.2)

The expressions of $\boldsymbol{\Lambda}^{(i)}(i=1,2,3)$ in (3.2) are given as follows. Let $q=$ $2 r+\left\{\left(r^{2}+r\right) / 2\right\}+K-1$ be the number of parameters or the number of the elements in $\boldsymbol{\theta}$. Then, we obtain

$\boldsymbol{\Lambda}^{(1)}=-\boldsymbol{\Lambda}^{-1}$ and $\left(\boldsymbol{\Lambda}^{(2)}\right)_{\cdot\{m(a b) l(c)\}}=\frac{2-\delta_{a b}}{2} \sum_{(a b)}^{2} \lambda^{\cdot a} \lambda^{b c} \quad(q \geq a \geq b \geq 1 ; c=1, \ldots, q)$,

where $(\cdot) \cdot\{m(a b) l(c)\}$ indicates the column of the matrix in parentheses corresponding to the product of the element $(\mathbf{M})_{a b}(q \geq a \geq b \geq 1)$ in $\mathbf{l}_{0}^{(2)}$ and $\left(\mathbf{l}_{0}^{(1)}\right)_{c},(\cdot)_{a b}$ is the $(a, b)$ th element of the matrix in parentheses, $(\cdot)_{c}$ is the $c$-th element of the vector in parentheses, $\delta_{a b}$ is the Kronecker delta, $\Sigma_{(a b)}^{2}$ is the sum of two terms considering the similar patterns in terms of $a$ and $b, \lambda^{c}$ is the $c$-th column of $\boldsymbol{\Lambda}^{-1}$, and $\lambda^{b c}=\left(\boldsymbol{\Lambda}^{-1}\right)_{b c}$.

Using the notations defined in similar manners, we obtain

$$
\begin{aligned}
& \left(\boldsymbol{\Lambda}^{(2)}\right)_{\cdot\{l(a) l(b)\}}=-\frac{1}{2} \boldsymbol{\Lambda}^{-1} \mathrm{E}\left(\frac{\partial^{3} \bar{l}}{\partial \boldsymbol{\theta}_{0}\left(\partial \boldsymbol{\theta}_{0}^{\prime}\right)^{<2>}}\right)\left(\lambda^{\cdot a} \otimes \lambda^{\cdot b}\right) \quad(a, b=1, \ldots, q), \\
& \left(\boldsymbol{\Lambda}^{(3)}\right)_{\cdot\{m(a b) m(c d) l(e)\}}=-\frac{1}{8}\left(2-\delta_{a b}\right)\left(2-\delta_{c d}\right) \sum_{(a b)}^{2} \sum_{(c d)}^{2}\left(\lambda^{\cdot a} \lambda^{b c} \lambda^{d e}+\lambda^{\cdot c} \lambda^{d a} \lambda^{b e}\right) \\
& (q \geq a \geq b \geq 1 ; q \geq c \geq d \geq 1 ; e=1, \ldots, q), \\
& \left(\boldsymbol{\Lambda}^{(3)}\right)_{\cdot\{m(a b) l(c) l(d)\}}=\frac{2-\delta_{a b}}{2} \sum_{(a b)}^{2}\left[\frac{1}{2} \lambda^{\cdot a}\left\{\boldsymbol{\Lambda}^{-1} \mathrm{E}\left(\frac{\partial^{3} \bar{l}}{\partial \boldsymbol{\theta}_{0}\left(\partial \boldsymbol{\theta}_{0}^{\prime}\right)^{<2>}}\right)\right\}_{b .}\left(\lambda^{\cdot c} \otimes \lambda^{\cdot d}\right)\right. \\
& \left.+\boldsymbol{\Lambda}^{-1} \mathrm{E}\left(\frac{\partial^{3} \bar{l}}{\partial \boldsymbol{\theta}_{0}\left(\partial \boldsymbol{\theta}_{0}^{\prime}\right)^{<2>}}\right)\left\{\left(\lambda^{\cdot a} \lambda^{b c}\right) \otimes \lambda^{\cdot d}\right\}\right] \\
& (q \geq a \geq b \geq 1 ; c, d=1, \ldots, q), \\
& \left(\boldsymbol{\Lambda}^{(3)}\right)_{\cdot\{m(a b c) l(d) l(e)\}}=-\frac{1}{2} \lambda^{\cdot a} \lambda^{b d} \lambda^{c e} \quad(a, b, c, d, e=1, \ldots, q) \\
& \operatorname{and}\left(\boldsymbol{\Lambda}^{(3)}\right) \cdot\{l(a) l(b) l(c)\} \\
& =-\frac{1}{2} \boldsymbol{\Lambda}^{-1} \mathrm{E}\left(\frac{\partial^{3} \bar{l}}{\partial \boldsymbol{\theta}_{0}\left(\partial \boldsymbol{\theta}_{0}^{\prime}\right)^{<2>}}\right)\left[\lambda^{\cdot a} \otimes\left\{\boldsymbol{\Lambda}^{-1} \mathrm{E}\left(\frac{\partial^{3} \bar{l}}{\partial \boldsymbol{\theta}_{0}\left(\partial \boldsymbol{\theta}_{0}^{\prime}\right)^{<2>}}\right)\left(\lambda^{\cdot b} \otimes \lambda^{\cdot c}\right)\right\}\right] \\
& +\frac{1}{6} \boldsymbol{\Lambda}^{-1} \mathrm{E}\left(\frac{\partial^{4} \bar{l}}{\partial \boldsymbol{\theta}_{0}\left(\partial \boldsymbol{\theta}_{0}^{\prime}\right)^{<3>}}\right)\left(\lambda^{\cdot a} \otimes \lambda^{\cdot b} \otimes \lambda^{\cdot c}\right) \quad(a, b, c=1, \ldots, q),
\end{aligned}
$$

where $(\cdot) \cdot\{m(a b c) l(d) l(e)\}$ denotes the column of the matrix in parentheses, corresponding to the product $\left\{\frac{\partial^{3} \bar{l}}{\partial\left(\boldsymbol{\theta}_{0}\right)_{a} \partial\left(\boldsymbol{\theta}_{0}\right)_{b} \partial\left(\boldsymbol{\theta}_{0}\right)_{c}}-\mathrm{E}\left(\frac{\partial^{3} \bar{l}}{\partial\left(\boldsymbol{\theta}_{0}\right)_{a} \partial\left(\boldsymbol{\theta}_{0}\right)_{b} \partial\left(\boldsymbol{\theta}_{0}\right)_{c}}\right)\right\} \frac{\partial \bar{l}}{\partial\left(\boldsymbol{\theta}_{0}\right)_{d}} \frac{\partial \bar{l}}{\partial\left(\boldsymbol{\theta}_{0}\right)_{e}}$.

In (A2), the expectations of the third and fourth derivatives are obtained by the Bartlett identity with the lower-order results shown in Ogasawara (2012, Section 1), e.g, 


$$
\begin{aligned}
\mathrm{E}\left(\frac{\partial^{3} \bar{l}}{\partial \boldsymbol{\theta}_{0} \partial \boldsymbol{\theta}_{0}^{\prime} \partial\left(\boldsymbol{\theta}_{0}\right)_{a}}\right)= & -\mathrm{E}\left(\frac{\partial^{2} l_{i}^{*}}{\partial \boldsymbol{\theta}_{0} \partial \boldsymbol{\theta}_{0}^{\prime}} \frac{\partial l_{i}^{*}}{\partial\left(\boldsymbol{\theta}_{0}\right)_{a}}\right)-\mathrm{E}\left(\frac{\partial^{2} l_{i}^{*}}{\partial \boldsymbol{\theta}_{0} \partial\left(\boldsymbol{\theta}_{0}\right)_{a}} \frac{\partial l_{i}^{*}}{\partial \boldsymbol{\theta}_{0}^{\prime}}\right) \\
& -\mathrm{E}\left(\frac{\partial l_{i}^{*}}{\partial \boldsymbol{\theta}_{0}} \frac{\partial^{2} l_{i}^{*}}{\partial \boldsymbol{\theta}_{0}^{\prime} \partial\left(\boldsymbol{\theta}_{0}\right)_{a}}\right)-\mathrm{E}\left(\frac{\partial l_{i}^{*}}{\partial \boldsymbol{\theta}_{0}} \frac{\partial l_{i}^{*}}{\partial \boldsymbol{\theta}_{0}^{\prime}} \frac{\partial l_{i}^{*}}{\partial\left(\boldsymbol{\theta}_{0}\right)_{a}}\right) .
\end{aligned}
$$

A.2 The equalities of the asymptotic cumulants in Theorem 2 and those of the statistics used by Lee and Poon (1986)

Recalling the definition of $\hat{\alpha}_{2}^{*}$ in $(3.10)$, we use a different notation $\hat{\alpha}_{2}^{* *}$ for the new estimator of $\alpha_{2}^{*}$ as:

$$
\hat{\alpha}_{2}^{* *}=-\left(\hat{\mathbf{H}}^{(1)} \hat{\mathbf{L}} \hat{\mathbf{H}}^{(1) \prime}\right)_{\eta \eta}=\alpha_{2}^{*}-\left(\hat{\mathbf{H}}^{(1)} \hat{\mathbf{L}} \hat{\mathbf{H}}^{(1) \prime}-\mathbf{H}^{(1)} \mathbf{\Lambda} \mathbf{H}^{(1) \prime}\right)_{\eta \eta} \equiv \alpha_{2}^{*}+m^{* *} .
$$

The result corresponding to (3.14) becomes

$$
\begin{aligned}
\mathrm{E}\left\{N\left(\mathbf{l}_{0}^{(1)}\right)_{a} m^{* *}\right\}= & -2\left(\frac{\partial \mathbf{H}^{(1)}}{\partial\left(\boldsymbol{\theta}_{0}\right)_{a}} \boldsymbol{\Lambda} \mathbf{H}^{(1) \prime}\right)_{\eta \eta}-\mathrm{E}\left[N \left\{\mathbf{H}^{(1)}\left(\frac{\partial^{2} \bar{l}}{\partial \boldsymbol{\theta}_{0} \partial \boldsymbol{\theta}_{0}^{\prime}}-\boldsymbol{\Lambda}\right) \mathbf{H}^{(1) \prime}\right.\right. \\
& \left.\left.+\mathbf{H}^{(1)} \frac{\partial^{3} \bar{l}}{\partial \boldsymbol{\theta}_{0}\left(\partial \boldsymbol{\theta}_{0}^{\prime}\right)^{<2>}}\left\{\left(\hat{\boldsymbol{\theta}}-\boldsymbol{\theta}_{0}\right) \otimes \mathbf{H}^{(1) \prime}\right\}\right\}_{\eta \eta} \frac{\partial \bar{l}}{\partial\left(\boldsymbol{\theta}_{0}\right)_{a}}\right]+O\left(N^{-1}\right) \\
= & -2\left(\frac{\partial \mathbf{H}^{(1)}}{\partial\left(\boldsymbol{\theta}_{0}\right)_{a}} \mathbf{\Lambda H}^{(1) \prime}\right)_{\eta \eta}-\left(\boldsymbol{\eta}^{(1) \prime}\right)^{<2>}\left[\mathrm{E}\left\{N \frac{\partial^{2} \bar{l}}{\left(\partial \boldsymbol{\theta}_{0}\right)^{<2>}} \frac{\partial \bar{l}}{\partial\left(\boldsymbol{\theta}_{0}\right)_{a}}\right\}\right. \\
& \left.+\mathrm{E}\left\{\frac{\partial^{3} \bar{l}}{\left(\partial \boldsymbol{\theta}_{0}\right)^{<2>} \partial\left(\boldsymbol{\theta}_{0}\right)_{a}}\right\}\right]+O\left(N^{-1}\right) \\
= & \mathrm{E}\left\{N\left(\mathbf{l}_{0}^{(1)}\right)_{a} m^{*}\right\}+O\left(N^{-1}\right),
\end{aligned}
$$

where the last equation is given by (3.14) and $\mathrm{E}\left\{N\left(\hat{\boldsymbol{\theta}}-\boldsymbol{\theta}_{0}\right)_{b} \frac{\partial \bar{l}}{\partial\left(\boldsymbol{\theta}_{0}\right)_{a}}\right\}=\delta_{b a}+O\left(N^{-1}\right)$. That is, it is seen that (3.14) and consequently Theorem 2 hold also for the pivots studentized by the ASE estimates of Lee and Poon (1986).

\section{REFERENCES}

Akahira, M., \& Takeuchi, K. (1981). Asymptotic efficiency of statistical estimators: Concepts and higher-order asymptotic efficiency. New York: Springer.

Akahira, M., \& Takeuchi, K. (1982). On asymptotic deficiency of estimators in pooled samples in the presence of nuisance parameters. Statistics and Decisions, 1, 17-38.

Amari, S. (1985). Differential-geometrical methods in statistics. Lecture notes in statistics. Vol.28. New York: Springer.

Bartlett, M.S. (1953). Approximate confidence intervals II. More than one unknown parameter. Biometrika, 40, 306-317.

Bhattacharya, R.N., \& Ghosh, J.K. (1978). On the validity of the formal Edgeworth expansion. The Annals of Statistics, 6, 434-451.

Bollen, K.A., \& Maydeu-Olivares, A. (2007). A polychoric instrumental variable (PIV) estimator for structural equation models with categorical variables. Psychometrika, 72, 309-326. 
Cox, N.R. (1974). Estimation of the correlation between a continuous and a discrete variable. Biometrics, 30, 171-178.

Hall, P. (1992a). The bootstrap and Edgeworth expansion. New York: Springer. Corrected printing, 1997.

Hall, P. (1992b). On the removal of skewness by transformation. Journal of the Royal Statistical Society, B, 54, 221-228.

Hannan, J.F., \& Tate, R.F. (1965). Estimation of the parameters for a multivariate normal distribution when one variable is dichotomized. Biometrika, 52, 664-668.

Hayakawa, T. (1977). The likelihood ratio criterion and the asymptotic expansion of its distribution. Annals of the Institute of Statistical Mathematics, 29, A, 359-378.

Jensen, J.L. (1989). Validity of the formal Edgeworth expansion when the underlying distribution is partly discrete. Probability Theory and Related Fields, 81, 507-519.

Jöreskog, K.G. (1990). New developments in LISREL: Analysis of ordinal variables using polychoric correlations and weighted least squares. Quality and Quantity, 24, 387-404.

Jöreskog, K.G. (1994). On the estimation of polychoric correlations and their asymptotic covariance matrix. Psychometrika, 59, 381-389.

Kraemer, H.C. (1981). Modified biserial correlation coefficients. Psychometrika, 46, 1981.

Lawley, D.N. (1956). A general method for approximating to the distribution of likelihood ratio criteria. Biometrika, 43, 295-303.

Lee, S.-Y., \& Poon, W.-Y. (1986). Maximum likelihood estimation of polyserial correlations. Psychometrika, 51, 113-121.

Lee, S.-Y., Poon, W.-Y., \& Bentler, P.M. (1989). Simultaneous analysis of multivariate polytomous variates in several groups. Psychometrika, 54, 63-73.

Lee, S.-Y., Poon, W.-Y., \& Bentler, P.M. (1990a). A three-stage estimation procedure for structural equation models with polytomous variables. Psychometrika, 55, 45-51.

Lee, S.-Y., Poon, W.-Y., \& Bentler, P.M. (1990b). Full maximum likelihood analysis of structural equation models with polytomous variables. Statistics and Probability Letters, 9, 91-97.

Lee, S.-Y., Poon, W.-Y., \& Bentler, P.M. (1992). Structural equation models with continuous and polytomous variables. Psychometrika, 57, 89-105.

Lee, S.-Y., Poon, W.-Y., \& Bentler, P.M. (1994). Covariance and correlation structure analyses with continuous and polytomous variables. In T.W. Anderson, K.T. Fang, \& I. Olkin (Eds.), IMS Lecture notes-Monograph series: Vol.24. Multivariate analysis and its applications (pp.347-358). Hayward, CA: Institute of Mathematical Statistics.

Lee, S.-Y., Poon, W.-Y., \& Bentler, P.M. (1995). A two-stage estimation of structural equation models with continuous and polytomous variables. British Journal of Mathematical and Statistical Psychology, 48, 339-358.

McCullagh, P. (1987). Tensor methods in statistics. London: Chapman \& Hall.

Muthén, B. (1984). A general structural equation model with dichotomous, ordered categorical, and continuous latent variable indicators. Psychometrika, 49, 115-132.

Muthén, B.O., \& Satorra, A. (1995). Technical aspects of Muthén's LISCOMP approach to estimation of latent variable relations with a comprehensive measurement model. Psychometrika, 60, 489-503.

Ogasawara, H. (2008). Inverse transformation of Hall's method back to Edgeworth type expansions: An expository note. In K. Shigemasu, A. Okada, T. Imaizumi, \& T. Hoshino (Eds.), New trends in psychometrics (pp.365-370). Tokyo: Universal Academy Press.

Ogasawara, H. (2010a). Accurate distribution and its asymptotic expansion for the tetrachoric correlation coefficient. Journal of Multivariate Analysis, 101, 936-948.

Ogasawara, H. (2010b). Asymptotic expansions for the pivots using log-likelihood derivatives with an application in item response theory. Journal of Multivariate Analysis, 101, 2149-2167.

Ogasawara, H. (2012). Supplement to the papers on "the polyserial correlation coefficients" 
and "discrepancy functions for general covariance structures". To appear in Economic Review (Otaru University of Commerce). http://www.res.otaru-uc.ac.jp/ ^hogasa/, http://barrel.ih.otaru-uc.ac.jp/.

Olsson, U., Drasgow, F., \& Dorans, N.J. (1982). The polyserial correlation coefficient. Psychometrika, 47, 337-347.

Pearson, K. (1909). A new method of determining correlation between a measured character $A$, and a character $B$, of which only the percentage of cases wherein $B$ exceeds (or falls short of) a given intensity is recorded for each grade of A. Biometrika, 7, 96-105.

Pearson, K. (1913). On the measurement of the influence of "broad categories" on correlation. Biometrika, 9, 116-139.

Poon, W.-Y., \& Lee, S.-Y. (1987). Maximum likelihood estimation of multivariate polyserial and polychoric correlation coefficients. Psychometrika, 52, 409-430.

Prince, B.M., \& Tate, R.F. (1966). Accuracy of maximum-likelihood estimates of correlation for a biserial model. Psychometrika, 31, 85-92.

Soper, H.E. (1913). On the probable error of the bi-serial expression for the correlation coefficient. Biometrika, 10, 384-390.

Stafford, J.E. (1992). Symbolic computation and the comparison of traditional and robust test statistics. Unpublished doctoral dissertation, University of Toronto, Canada.

Stroud, A.H., \& Secrest, D. (1966). Gaussian quadrature formulas. Englewood Cliffs, NJ: PrenticeHall.

Stuart, A., \& Ord, J.K. (1994). Kendall's advanced theory of statistics: Distribution theory (6th ed., Vol.1). London: Arnold.

Taniguchi, M., \& Kakizawa, Y. (2000). Asymptotic theory of statistical inference for time series. New York: Springer.

Taniguchi, M., \& Watanabe, Y. (1994). Statistical analysis of curved probability densities. Journal of Multivariate Analysis, 48, 228-248.

Tate, R.F. (1955a). The theory of correlation between two continuous variables when one is dichotomized. Biometrika, 42, 205-216.

Tate, R.F. (1955b). Applications of correlation models for biserial data. Journal of American Statistical Association, 50, 1078-1095.

Viraswami, K., \& Reid, N. (1996). Higher-order asymptotics under model misspecification. Canadian Journal of Statistics, 24, 263-278.

(Received January 11 2011, Revised June 6 2011) 